\title{
Building giant-planet cores at a planet trap
}

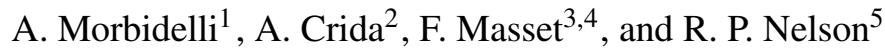 \\ 1 Observatoire de la Côte d'Azur, BP 4229, 06304 Nice Cedex 4, France \\ e-mail: morby@obs-nice.fr \\ 2 Institut für Astronomie \& Astrophysik, Univ. of Tubingen, Germany \\ 3 Laboratoire AIM, CEA/Saclay, France \\ ${ }^{4}$ IA-UNAM, Mexico City, Mexico \\ 5 Queen Mary, University of London, UK
}

Received 24 August 2007 / Accepted 5 November 2007

\begin{abstract}
Context. A well-known bottleneck for the core-accretion model of giant-planet formation is the loss of the cores into the star by type I migration, due to the tidal interactions with the gas disk. It has been shown that a steep surface-density gradient in the disk, such as the one expected at the boundary between an active and a dead zone, acts as a planet trap and prevents isolated cores from migrating down to the central star.

Aims. We study the relevance of the planet trap concept for the accretion and evolution of systems of multiple planetary embryos/cores. Methods. We performed hydrodynamical simulations of the evolution of systems of multiple massive objects in the vicinity of a planet trap. The planetary embryos evolve in 3 dimensions, whereas the disk is modeled with a 2D grid. Synthetic forces are applied onto the embryos to mimic the damping effect that the disk has on their inclinations.

Results. Systems with two embryos tend to acquire stable, separated and non-migrating orbits, with the more massive embryo placed at the planet trap and the lighter one farther out in the disk. Systems of multiple embryos are intrinsically unstable. Consequently, a long phase of mutual scattering can lead to accreting collisions among embryos; some embryos are injected into the inner part of the disk, where they can be evacuated into the star by type I migration. The system can resume a stable, non-migrating configuration only when the number of surviving embryos decreases to a small value $(\sim 2-4)$. This can explain the limited number of giant planets in our solar system. These results should apply in general to any case in which the type I migration of the inner embryo is prevented by some mechanism, and not solely to the planet trap scenario.
\end{abstract}

Key words. Solar system: formation - planets and satellites: general

\section{Introduction}

The core-accretion model for the formation of giant planets meets a serious problem. The cores of the planets, embedded in the gas disk, should undergo type I migration towards the central star (Ward 1997; Tanaka et al. 2002). The migration rate increases linearly with the core's mass. For masses of order of a few Earth masses, the timescale for the engulfment of the core into the star is much shorter than that for the accretion of a massive atmosphere and the transition to the status of a giant planet (Pollack et al. 1996). Even the most recent models of giantplanet formation, accounting for the positive feedback of migration on accretion (Alibert et al. 2005), require type I migration rates that are at least 10 times slower than estimated by analytic theory and measured in hydrodynamical simulations for cores embedded in laminar disks.

The situation is very different in turbulent disks (Nelson \& Papaloizou 2003; 2004; Nelson 2005; Johnson et al. 2006). The large scale fluctuations of the disk surface density under MRI turbulence exert a stochastic torque onto the core. Consequently, the evolution of the core's semi major axis resembles a random walk. For at least some of the cores, the dynamical lifetime against collisions with the star can increase substantially relative to the non-turbulent case, dominated by type I migration (Nelson 2005). However, the problem is that the stochastic torque exerted by turbulent fluctuations also excites the orbital eccentricities of cores and planetesimals. This inhibits accretion because mutual planetesimal collisions become disruptive and runaway growth (which is effective only when the core's escape velocity is large relative to the velocity dispersion of the planetesimal population) is triggered only for bodies more massive than about Ceres or Pluto. Thus, we are confronted with a conundrum. If we invoke turbulence to solve the core's type I migration problem, we cannot grow the core in first place; if we invoke the core's growth in a low-turbulent portion of the disk (the so-called "dead zone"; Gammie 1996; Stone et al. 1998), then we cannot avoid type I migration.

Some possibilities have been proposed in order to avoid type I migration in non-turbulent (e.g. laminar) disks. Masset et al. (2006b) found that, due to non-linear effects, the migration rate is strongly reduced (or even halted) for a core of about 10-20 Earth masses $\left(M_{\oplus}\right)$, the exact value depending on disk parameters. Paardekooper \& Mellema (2006) found that inward type I migration could be reduced or reversed in non-isothermal discs, due to changes in the gas density in regions leading and trailing the planet.

The "planet trap" concept, proposed by Masset et al. (2006a), provides an alternative, appealing possibility, that we explore more in detail in this paper. The planet trap results from the balance between the (negative) differential Lindblad torque, usually responsible for type I migration (Ward 1997), and the (positive) 
coorbital corotation torque that is exerted if the planet is in a portion of the disk characterized by a steep and positive density gradient in the radial direction. The name "planet trap" comes from the fact that planetary embryos, migrating towards the central star, stop when they encounter the aforementioned density gradient, as if they were captured into a trap (see figures in Masset et al. 2006a or Fig. 2 in this work). Notice that a positive density gradient in the disk also acts as a trap for small planetesimals suffering gas-drag. In fact, elsewhere in the disk the gas orbits around the star with sub-Keplerian speed because of a negative pressure gradient. This forces the planetesimals to drift inwards. However, at a positive density gradient, the pressure gradient is also positive, so that the gas is super-Keplerian. Consequently, planetesimals migrating inward because of gas drag would get trapped at the point where the rotation profile transitions between sub and super-Keplerian values. This would clearly help the buildup of a large core located at/near the planet trap.

A density gradient responsible for the onset of a planet trap is expected to exist at the transition between the inner, turbulent portion of the disk and the dead zone, because the dead zone is characterized by a smaller transport coefficient (or turbulent viscosity). This opens the intriguing possibility that giant-planet cores form in the dead zone (where the dispersion velocities are low and accretion can be effective) but are not lost by type I migration because of the planet trap at the inner edge of the dead zone.

The location of the inner edge of the dead zone is very uncertain. Models of disk chemistry (see for instance Ilgner \& Nelson 2006a) suggest that only the region interior to $0.2 \mathrm{AU}$ is active (due to thermal ionization of potassium), with the dead zone lying outside this region. However, models accounting for the diffusion of free charges from the upper, ionized layers of the disk down to the mid-plane (Ilgner \& Nelson 2006b; Turner et al. 2007) suggest that the magneto-rotational instability fills the whole thickness of the disk up to at least $5 \mathrm{AU}$, under favourable circumstances. Moreover, Chiang \& Murray-Clay (2007) argued that the boundary between the active zone and the dead zone moves outward with time. A gas density gradient suitable for the creation of a planet trap could also be caused by the partial depletion of the inner few AUs of the disk by winds driven by the magnetic field (Ferreira et al. 2006). Notice that there is a growing body of evidence for the existence of transition disks with inner cavities of several AUs in size (see for instance Forrest et al. 2004; Calvet et al. 2004, 2005; Bergin et al. 2004; Pietu et al. 2006). So, the location of the planet trap is an open issue, but it is not excluded that it can be several AUs away from the central star. By considering that the planet trap might have played a role in the formation of the cores of the giant planets of our solar system, we implicitly assume that it was located at about 5 AU.

The location and effectiveness of the planet trap depend on the disk properties, but are the same for a large variety of embryo's masses. Thus, all planetary embryos tend to go to the same location (see for instance Fig. 3 by Masset et al. 2006a). Masset et al. conjectured that, for this reason, the planet trap might be a sweet spot for the rapid accretion of a massive planetary core from mutual collisions of embryos. However, they did not test this conjecture with numerical simulations. Actually, Fig. 3 by Masset et al. is very misleading. The figure superposes the evolution of many embryos of multiple masses, each of which integrated separately. Neither direct nor indirect perturbations among the embryos were taken into account. Thus, the clustering of embryos at the planet trap can be artificial.
Terquem \& Papaloizou (2007) took the opposite attitude concerning the relevance of the planet trap. They argued that, in a multi-embryo system, embryo-embryo scattering could move the semi major axis of an embryo across the density gradient into the inner disk, where type I migration would resume again. Moreover they speculated that the interaction among the embryos at the vicinity of the trap would excite their orbital eccentricities; embryos on eccentric orbits would only sample the density gradient for a fraction of the orbit and, consequently, they would feel a reduced corotation torque.

In summary, the actual role of the planet trap for the accretion and preservation of giant-planet cores from a system of multiple planetary embryos is not clear, and different outcomes can be legitimately expected. The purpose of this paper is to conduct specific hydrodynamical simulations of the dynamics of massive bodies in the vicinity of the planet trap. In Sect. 2 we start by presenting the numerical scheme that we use in the simulations and in Sect. 3 we describe our set-up for building the required surface density gradient and identifying the location of the planet trap. We then proceed in sequence, making the problem more complex step by step. In Sect. 4 we investigate the dynamics of two planetary embryos, initially on distant orbits, as a function of their masses and mass ratio. In Sect. 5, we study the dynamics of two embryos on unstable orbits, scattering off each other. Finally, in Sect. 6, we consider a system with many (i.e. 10) planetary embryos, initially of equal masses.

Several simulations of the dynamics of multiple protoplanets in a gas disk have been presented in the literature. Papaloizou \& Szuszkiewicz (2005) studied the the migrationinduced resonance trapping in a system of two planets with masses in the Earth mass range. McNeil et al. (2005), Cresswell \& Nelson (2006) and Terquem \& Papaloizou (2007) studied the migration of multiple proto-planetary embryos. These studies used either $\mathrm{N}$-body integrators with fake forces that mimic the planet-disk interactions (McNeil et al. 2005; Terquem \& Papaloizou 2007) or fully hydrodynamical simulations (Papaloizou \& Szuszkiewicz 2005) or both (Cresswell \& Nelson 2006). In all cases, a classical laminar disk was considered, without a planet trap. The general result is that, after an initial phase during which some mutual collisions may be possible, the protoplanets find a relative configuration that is stable, each object locked in resonance with another. The full system collectively evolves by type I migration towards the central star. The difference between our results and this general evolution will enlighten the role of the putative planet trap.

\section{An hybrid numerical simulation scheme}

Because some of our simulations will involve mutual scattering and collisions among planetary embryos, it is important that the simulations are done in three dimensions, namely allowing the orbits to evolve also in inclination. In fact, it is well known that in a co-planar system the ratio between collisions and scattering events is artificially large (Chambers 2001), which invalidates the first generation of planet accretion simulations done in two dimensions. However, doing a fully three-dimensional simulation of the evolution of the embryos and the disk would be prohibitively expensive, from the computational point of view. For this reason we have adopted a compromise approach, where the embryos are allowed to evolve in three dimensions, the disk is simulated with a two-dimensional grid, and fake damping forces are applied on embryo inclinations.

Our hybrid $(2 \mathrm{D}+3 \mathrm{D})$ implementation is built on the code FARGO by Masset $(2000 a, b)$. Each massive body is 
identified by its three dimensional positions $(x, y, z)$ and velocities $\left(v_{x}, v_{y}, v_{z}\right)$. As the disk is planar, each cell is identified by the position of its center $\left(x_{\mathrm{c}}, y_{\mathrm{c}}\right)$. The potential of the body-cell gravitational interaction is therefore:

$U=-\frac{G M m}{\sqrt{\left(x-x_{\mathrm{c}}\right)^{2}+\left(y-y_{\mathrm{c}}\right)^{2}+z^{2}+\epsilon^{2}}}$,

where $G$ is the gravitational constant (assumed to be 1 ), $M$ is the mass of the body (in solar masses), $m$ is the mass of the gas in the cell and $\epsilon$ is the so-called smoothing parameter, here assumed to be $70 \%$ of the local thickness of the disk $(H / r=$ 0.05 in all simulations presented in this paper). The smoothing parameter is intended to mimic the thickness of the disk and not the inclination of the planet. The functional form (1) has been chosen in order to have an analytic function that tends to the usual potential adopted in 2D simulations for vanishing $z$, and to the correct Newtonian potential for $z \gg \epsilon$. Moreover, because the smoothing parameter that we adopt is larger than the Hill radius and the Bondi radius of the embryos, we account for the torque exerted on the planet by every cell of disk (e.g. with no torque cut-off; see Masset et al. 2006b for a discussion).

With this potential, the planetary bodies suffer migration and eccentricity damping, but the inclination is not affected because the disk cannot support vertical waves. In reality, the disk also damps the planet's orbital inclination (Lubow \& Ogilvie 2001). Thus, following Tanaka \& Ward (2004), we mimic this damping effect by exerting an acceleration on the planet's $v_{z}$ equal to

$F_{\text {damp }, z}=M\left(\frac{\Sigma_{\mathrm{g}}}{c_{\mathrm{s}}^{4}}\right) \Omega\left(2 A_{z}^{\mathrm{c}} v_{z}+A_{z}^{\mathrm{s}} z \Omega\right)$

where $\Sigma_{\mathrm{g}}$ is the mean surface density of the gas on an annulus located at the planet's current radial distance, $c_{\mathrm{s}}$ is the local sound speed and $\Omega$ is the Keplerian angular velocity. The constants $A_{z}^{\mathrm{c}}$ and $A_{z}^{\mathrm{s}}$ are given by Tanaka \& Ward (2004). Tanaka \& Ward's theory is linear, and valid for small inclinations (and eccentricities). More recently, Cresswell et al. (2007) studied the damping of eccentricity and inclination with 3D numerical simulations and found that, for large initial values of $e$ and/or $i$, the damping is slower than predicted by the linear theory. However, the similarity between the damping rates of $e$ and $i$ still holds. Thus, here we tune empirically the coefficients $A_{z}^{\mathrm{c}}$ and $A_{z}^{\mathrm{s}}$ in (2) by a multiplicative factor, so that the resulting damping on the inclination occurs in most occasions on the same timescale of the damping of the eccentricity, which is self-consistently computed by our hydrodynamical code. After several tests, the multiplicative factor was set equal to 0.1 (i.e. Tanaka and Ward formula was divided by a factor of 10). As an example, Fig. 1 shows the resulting evolution of the eccentricity and of the inclination after two scattering events in one simulation that will be described in Sect. 6. With this choice of the multiplicative factor, the damping timescales of both quantities essentially coincide. We acknowledge that, for small inclinations, the real damping rate of the inclination should be faster than the one that we impose. A faster inclination damping rate would increase somewhat the probability that embryos undergo mutual collisions, in particular in the cases where the scattering phase is protracted (as in the multiembryo simulation of Sect. 6). Because in this paper we do not intend to study the planet accretion rate from a quantitative point of view, but rather to investigate the qualitative aspects of the dynamics of systems of embryos in the vicinity of a planet trap, we think that having inclination damping rates that might be smaller in some cases than the real ones should not be a severe limitation.

As in FARGO, the gravitational interactions among the massive bodies and their interactions with the star are integrated with

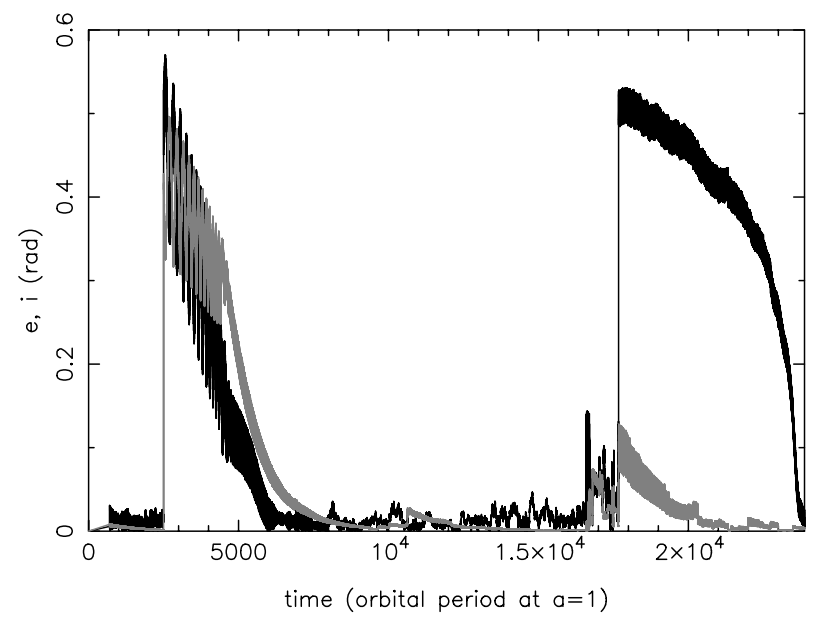

Fig. 1. The evolution of the eccentricity (black curve) and inclination (in radians; gray curve) of an embryo undergoing scattering from other objects in the system. Notice the good agreement between the damping timescales of eccentricity and inclination after each major scattering event.

a fixed time-step Runge Kutta integrator (Steiner 1996) of 5 th order. The choice of a fixed time-step is quite mandatory in the architecture of the code, and, in principle, it may fail to give an accurate evolution in case of very close encounters among the embryos. However, the time-step, imposed by the so-called CFL condition (Masset 2000a) for the correct simulation of the gas dynamics, is very small, of order of $1 / 180$ of the orbital period at our unit of distance $(a=1)$, so that the simulation should be correct in most cases. Again, we stress that we are more interested in this work on the qualitative dynamics of embryos in the vicinity of the planet trap, rather than in its quantitative aspects (which would not be accurate anyway given the limitations of the two dimensional treatment of the gas disk).

Our implementation of the Runge Kutta algorithm searches for physical collisions among the embryos. This is done by computing, at each time-step, the hyperbolic arc of an embryo relative to each other one, and by confronting the minimal approach distance along this arc with the physical sizes of the bodies. For the physical radii, we assume for simplicity that the bulk density of the objects is $2 \mathrm{~g} / \mathrm{cm}^{3}$ and that our unit distance is the astronomical unit. If the unit of distance or the bulk density were larger (smaller), the physical collisions would result less (more) frequent than in our simulations. We assume that all collisions are accretional. When two embryos collide, one embryo is suppressed from the simulation, while the other one acquires the sum of the masses and its velocity is changed so that the linear momentum of the two colliding embryos is carried by the surviving one.

\section{Simulation set up}

In all our simulations, the disk extends from 0.4 to 3 in radius, and the $2 \mathrm{D}$ grid has resolution $260 \times 450$ in radius and azimuth, respectively. The disk aspect ratio is $5 \%$ at all radii. To set a surface density profile with a positive gradient around $a=1$ we proceed as follows (see also Masset et al. 2006a). The viscosity of the disk is set as a function of radius. More precisely, inside $r=0.9$ the viscosity $v$ is given with an $\alpha$ prescription (Shakura $\&$ Sunyaev 1973), with $\alpha=6 \times 10^{-3}$. Between $r=0.9$ and $r=1.1$, the viscosity decreases linearly as

$v=v(r=0.9) \times(4.857-r \times 4.2857)$. 

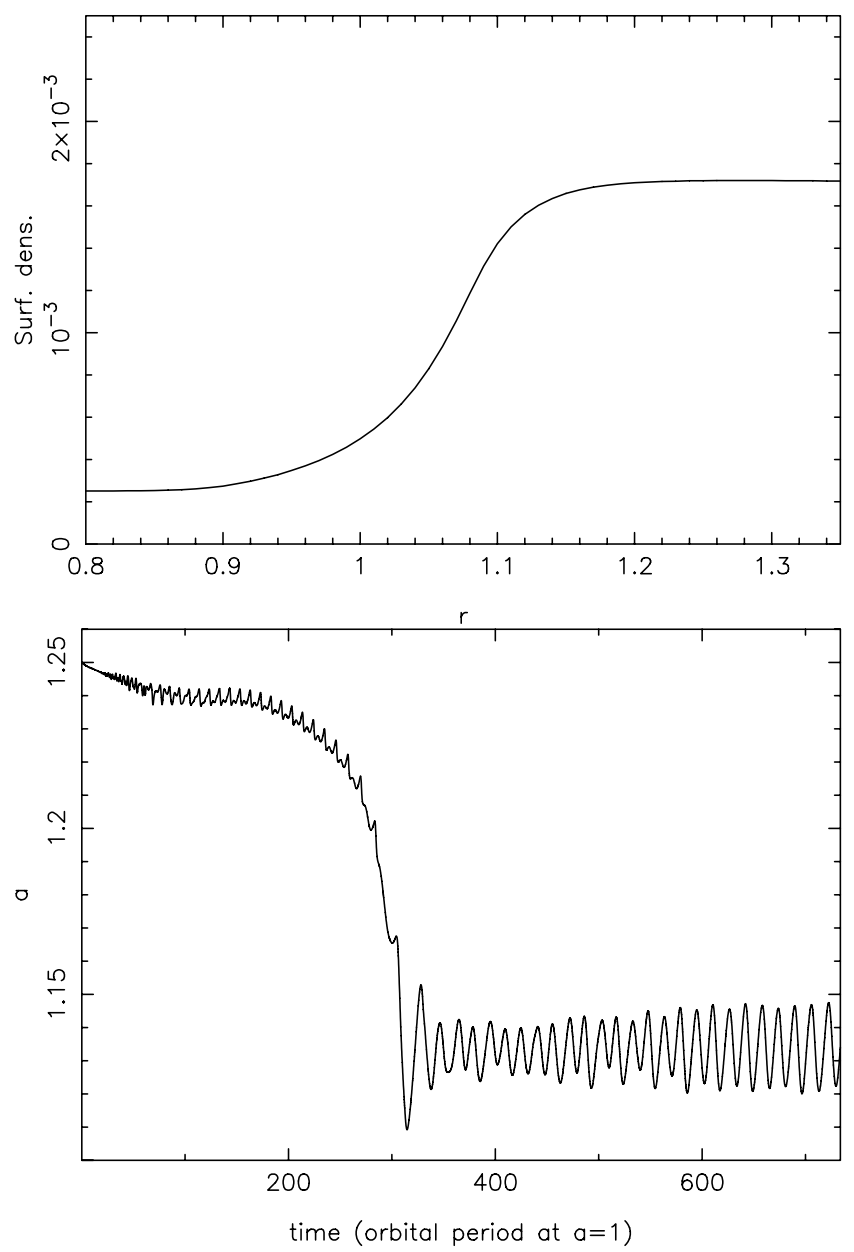

Fig. 2. Top: the equilibrium surface density distribution of the disk, with the steep surface density gradient at $r=1$, obtained with the viscosity and boundary conditions prescription illustrated in the text. Bottom: the evolution of the semi major axis of a $10 M_{\oplus}$ object, in the disk shown on the left panel. The object is rapidly captured into the planet trap. This simulation is used to determine the location of the trap, for the subsequent runs.

In the disk beyond $r=1.1$, the viscosity is given again with an $\alpha$ prescription, but with $\alpha$ decreased by a factor $4.857-1.1 \times$ $4.2857=0.143$ with respect to the inner disk .

In a first simulation, we let the disk evolve for 15000 orbits at $a=1$, without any planetary body in it. The initial surface density distribution increases linearly from $2.5 \times 10^{-4}$ at $r=0.4$ to $1.7 \times 10^{-3}$ at $r=3$ in the units specified above. The boundary conditions preserve the surface density at the border of the grid, and act as sources or sinks of mass. Under this setting and the viscosity prescription described above, the disk evolves rapidly to a new equilibrium configuration, illustrated in top panel of Fig. 2, that exhibits the desired surface density gradient.

We then use the final gas distribution of this simulation as an input for a new simulation, in which we release a $10 M_{\oplus}$ core at $r=1.25$, with a small (e.g. $\sim 10^{-3}$ ) eccentricity and a null inclination. The core evolves inward rapidly and gets caught at the planet trap (see bottom panel of Fig. 2). Notice the acceleration of the inward migration, as the core approaches the trap, already discussed by Masset et al. (2006a). Also, the evolution of the semi major axis is not monotonic, but presents large oscillations, that become very pronounced after the capture in the trap. This is due to the existence of a vortex, produced by a Rossby instability at the summit of the surface density gradient, where the vortensity has a local maximum (Masset et al. 2006a). The planet trap is located inside the orbital radius of the vortex, at a distance equal to a few times $H / r$. Thus, the synodic period between the core and the vortex is of order $P \times r / H$, where $P$ is the orbital period, and corresponds to the oscillation period visible in Fig. 2.

Because the planet trap mechanism is independent of the planet's mass, in the simulations of the following sections, whenever we need to place a planetary embryo in the trap, we simply adopt as initial conditions the final output of the simulation in the bottom panel of Fig. 2 and just reset the mass of the body.

\section{Dynamics of two embryos on initially separated orbits}

Our first set of simulations accounts for two planetary embryos, the more massive of which is initially placed at the trap. The lighter embryo is placed initially at $a=1.35$, on a quasi-circular orbit $\left(e \sim 10^{-3}\right)$ with an initial inclination of $\sim 10^{-2}$ radians. We have done three simulations, with masses of the embryos pairs equal to 10 and $5 M_{\oplus}, 5$ and $2.5 M_{\oplus}, 2$ and $1 M_{\oplus}$. Figure 3 shows two examples of evolution, from the first and the last simulation. The outer object is represented by three curves, denoting its perihelion distance, semi major axis and aphelion distance, respectively, so that the evolution of the eccentricity can also be captured by eye (when the orbit is circular the three curves are superposed). For the inner embryo, only the semi major axis is plotted versus time.

In all cases we observe that, after some inward migration, the outer embryo stops. In none of the cases the outer embryo reaches the planet trap. Thus, neither the conjecture by Masset et al. (2006a) - namely that embryos cluster at the planet trap and accrete with each other - nor that by Terquem \& Papaloizou (2007) - that embryos scatter each other off the trap, or become eccentric and insensitive to the planet trap mechanism - appears to be true.

The mechanism that stops the inward migration of the outer embryo depends on the embryo masses. If the embryos are quite small as in the 2-1 $M_{\oplus}$ simulation (as well as in the 5-2.5 $M_{\oplus}$ one, not illustrated here), the outer embryo is trapped in a mean motion resonance with the inner one (the 4:5 resonance for the case illustrated in the top panel of Fig. 3, for $1000<t<6000$ ). When resonance trapping occurs, the eccentricity of the outer embryo is enhanced to a non-zero equilibrium value. The direct perturbation of the inner embryo onto the outer one is crucial in this dynamical evolution. To illustrate this, after 6050 orbits (time marked by the leftmost vertical dashed line on the top panel of Fig. 3), we suppress the direct perturbations between the embryos. Immediately, the inward migration of the outer embryo starts again, and the eccentricity is rapidly damped. After 6200 orbits (time marked by the rightmost vertical dashed line on the top panel of Fig. 3), we switch on again the direct perturbations between the embryos. Then, the outer embryo is captured into the next mean motion resonance (the 5:6 resonance), which leads to eccentricity excitation again.

Conversely, if the embryos are massive, as in the $10-5 M_{\oplus}$ simulation, the outer embryo is repelled by the inner one through indirect perturbations, that is through the modifications in the disk surface density created by the first embryo. To demonstrate the dominant role of the indirect perturbation, again we suppress the direct perturbations between the embryos after 4200 orbital periods (time marked by the vertical dashed line in the bottom panel of Fig. 3). The evolution of the bodies does not change 

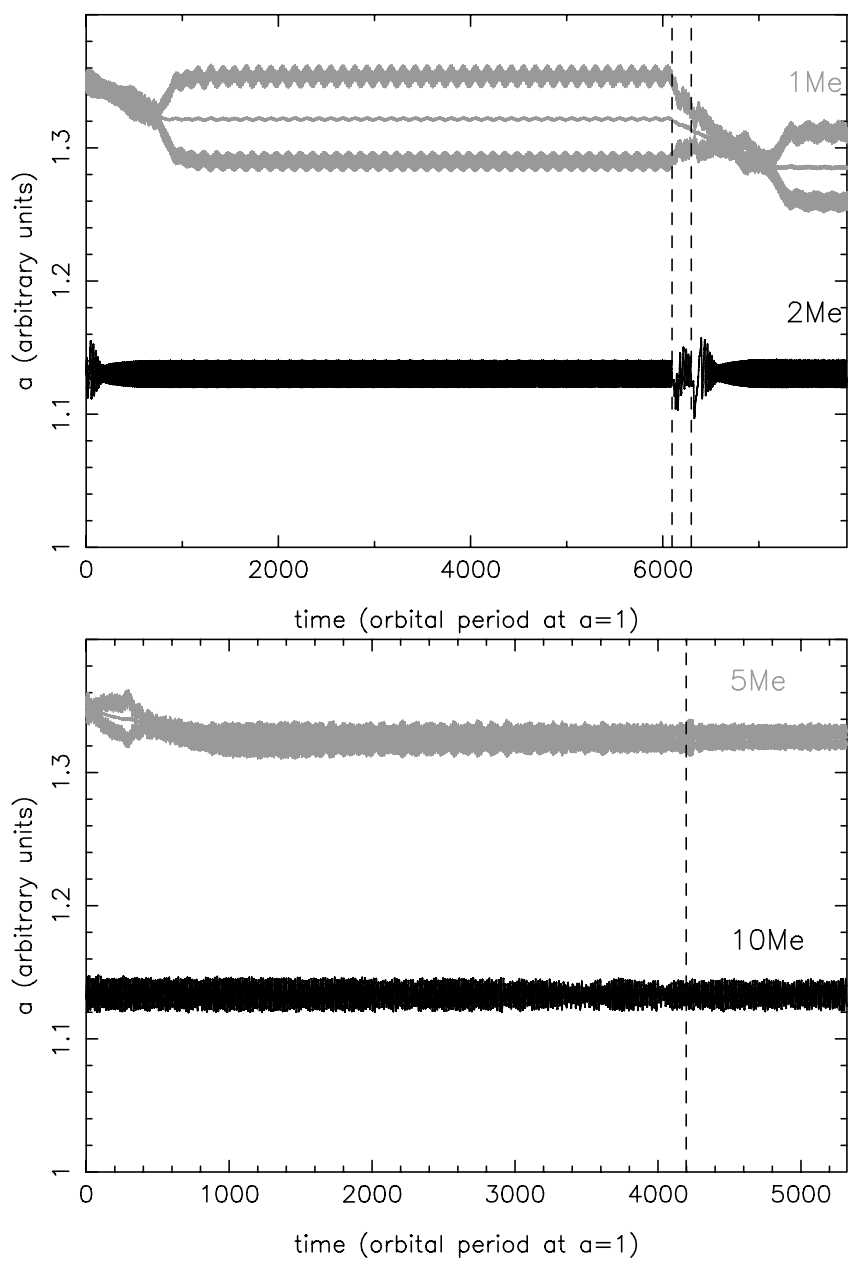

Fig. 3. The evolution of two embryos in presence of a planet trap. The outer body is represented by three curves, denoting perihelion distance, semi major axis and aphelion distance of its orbit. The inner embryo is initially placed at the trap. Because its eccentricity remains small only the evolution of its semi major axis is plotted. Top panel: the masses of the inner and outer embryos are 2 and $1 M_{\oplus}$ respectively. Bottom panel: the masses of the inner and outer embryos are 10 and $5 M_{\oplus}$ respectively. The vertical dashed lines show the times at which the direct perturbations between the embryos are switched off and on, respectively. See text for discussion.

significantly in this case. Notice also that the eccentricity of the bodies remains small all the time. Thus, the outer embryo is not captured in a mean motion resonance with the inner one.

To investigate which kind of indirect perturbation repels the outer embryo in this case, we compare in Fig. 4 the surface density profile of the disk in this simulation (black curve) with the one obtained in the $2-1 M_{\oplus}$ simulation (gray curve). In the case of the $2-1 M_{\oplus}$ simulation, the surface density profile is essentially the unperturbed one, shown on the top panel of Fig. 2. The little "bump" visible at $r \sim 1.2$ is due to the aforementioned vortex. The outer embryo is in a region of the disk where the surface density profile is flat, so that it would suffer type I migration unless it is restrained by a resonance with the inner embryo. In the case of the $10-5 M_{\oplus}$ simulation, the surface density profile is strongly modified. The "bump" due to the vortex is more pronounced. Also, the torque exerted by the inner embryo on the outer disk forces the surface density to acquire a positive gradient at equilibrium in the $1.2-1.8$ radial range. This produces a new planet trap, in which the outer embryo is eventually captured.

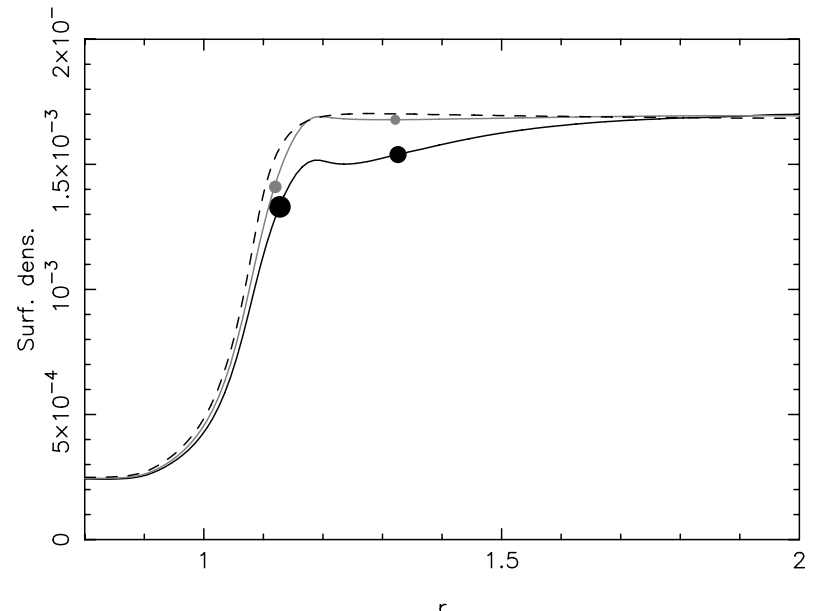

Fig. 4. Radial profiles of the azimuth-averaged disk surface densities in the case of the 10-5 $M_{\oplus}$ simulation (black curve), of the 2-1 $M_{\oplus}$ simulation (gray curve) and in absence of embryos (dashed curve). The radial location of each embryo is reported with a filled circle, with size proportional to the object's physical radius. The ordinate of each circle is arbitrary, and has been chosen so to please each embryo on the corresponding surface density curve, for illustrative purposes.

In all the simulations described above, the inclination of the outer embryo damps continuously and exponentially, down to values of order $10^{-4}$ radians or smaller. This is due to the fact that close encounters with the inner embryo do not occur, and that neither mean motion resonance trapping nor the indirect perturbations excite the inclination. Consequently, the damping effect exerted by the disk, forced through the prescription (2), dominates the evolution of the inclination.

The lesson that we derive from these experiments is that, at least in these configurations, the planet trap is effective to prevent type I migration of a system of two embryos. Close approaches and collisions between embryos do not occur. Thus, the growth of the embryos can be due only to the sole accretion of small planetesimal, as in a classical oligarchic growth mode (Kokubo $\&$ Ida 1998). The case of a reversed mass ratio between the embryos (i.e. the case where the outer one is the more massive) will be discussed in the second part of the next section.

All the simulations illustrated above have been conducted in the framework of a laminar disk. It is interesting to investigate how the embryos' dynamics can be affected by large scale turbulent fluctuations in the disk. Strong turbulence might in principle destroy the planet trap mechanism, or inhibit resonant trapping and isolation of the two massive bodies. Turbulence is expected to arise in the disk due to Magneto-RotationalInstabilities (MRI; e.g. Balbus \& Hawley 1991). Full MHD simulations are beyond the scope of this paper, but we can mimic the effect of MRI turbulence adding a stochastic planar torque of suitable amplitude on the embryos in our laminar hydrodynamical simulations. The recipe that we follow for the generation of the stochastic torque is that implemented by Ogihara et al. (2007; see formulae 5-7 in that paper). In turn, Ogihara et al. implementation is based on the scheme proposed in Laughlin et al. (2004), which was calibrated on the results of MHD simulations. Notice, however, that MRI turbulence does not only induce fluctuations in the gas density distribution, but also in the gas velocity field. These velocity fluctuations may affect the horseshoe streamlines in the corotation region (Papaloizou et al. 2004), and therefore the magnitude of the corotation torque. Consideration of this effect goes beyond the scope of this paper, so the fluctuations in 


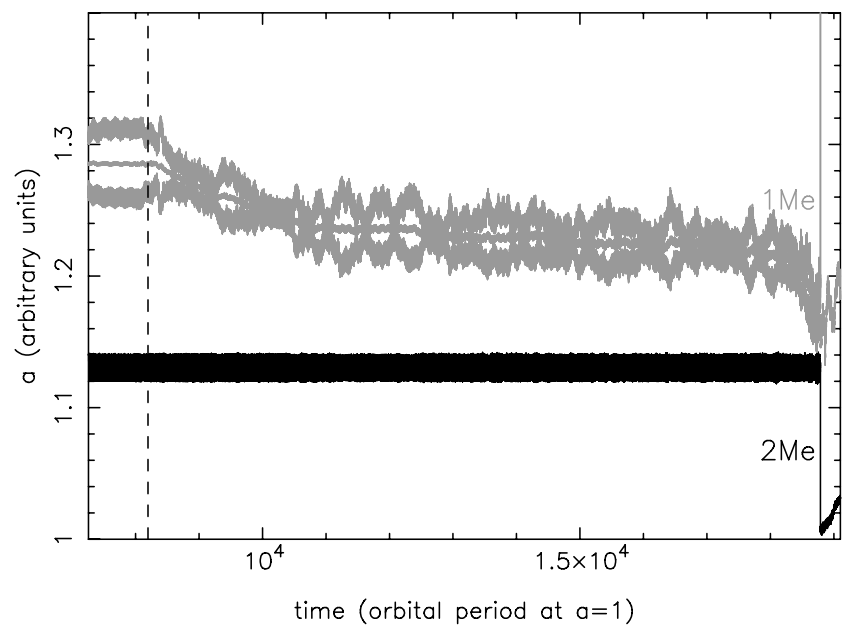

Fig. 5. The continuation of the simulation in the top panel of Fig. 3, but adding the effects of turbulent fluctuations in the gas surface density distribution of relative amplitude of about $1 \%$. The vertical dashed line marks the time when the stochastic (turbulent) torque is switched on.

the velocities are neglected in these approximate implementations of the stochastic torque.

We have continued the simulation presented in the top panel of Fig. 3, implementing the stochastic torque on both embryos. We have done three simulations, with $\gamma=10^{-1}, 10^{-2}$ and $10^{-3}$, where $\gamma$ is the dimensionless parameter identified by Ogihara et al. as indicator of the "turbulence strength", corresponding approximately to the relative amplitude of the fluctuations of the gas density field (Fig. 5 presents the result for $\gamma=10^{-2}$ ). In all cases, the stochastic torque does not destroy the planet trap mechanism. The inner embryo keeps oscillating around $a=1.13$. This is in agreement with the similar tests performed by Masset et al. (2006a), although with a simpler recipe for the stochastic torque. The trapping of the outer embryo in the mean motion resonance is inhibited in the cases with $\gamma=10^{-1}$ and $10^{-2}$. In fact, the embryo leaves the 5:6 resonance as soon as the stochastic torque is switched on, and is not trapped permanently in any other mean motion resonance. Thus, it migrates stochastically towards the inner embryo, until it has a close encounter with it. In the example of Fig. 5 the close encounter scatters the inner embryo into the inner part of the disk, whereas the lighter embryos is scattered onto an orbit with much larger semi major axis and eccentricity (see however Sect. 5 for a more detailed discussion of the outcome of embryo-embryo scattering). In the case of reduced turbulence strength $\left(\gamma=10^{-3}\right)$, however, the outer embryo is not released from the resonance. We do not show a figure for this case, because the evolution of the system is indistinguishable from that shown in the top panel of Fig. 3.

The MHD simulations of Laughlin et al. (2004) suggest that $\gamma$ should be of order $10^{-3}$ to $10^{-2}$ in the active zone of the disk, although large uncertainties exist. In the dead zone, however, the turbulence strength should be much smaller. Recall that the planet trap should be positioned at the transition between the active and the dead zone. Thus, the outer embryo, which is beyond the planet trap location, should evolve in the dead zone. Although waves generated by the density fluctuation in the live zone can propagate into the dead zone, we expect that the stochastic perturbations on the outer embryo have a reduced intensity. Therefore, from the experiments above, we expect that it is unlikely that the outer embryo could escape trapping in mean motion resonances with the inner embryo. Notice also that the strength of mean motion resonances scales with the square root

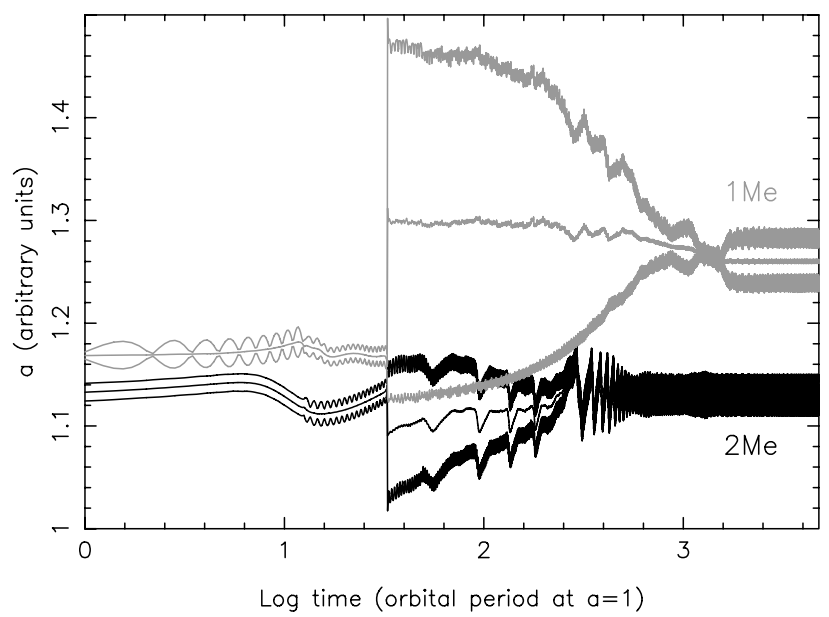

Fig. 6. The evolution of two embryos on initially close, unstable orbits, in the vicinity of a planet trap. Each body is represented by three curves, denoting perihelion distance, semi major axis and aphelion distance of its orbit. The time is plotted in logarithmic scale in order to enlighten the early phase of mutual scattering between the two embryos.

of the embryo masses so that, for more massive embryos, the isolation mechanism should be even more robust against the effects of turbulence.

\section{Dynamics of two embryos on unstable, scattering orbits}

If the embryos are locked into a mutual mean motion resonance, their orbital separation remains constant. However, if at the same time the embryos are growing by accretion of planetesimals, they can become too massive for their orbital separation to be stable. In fact, the minimal orbital separation that allows stability depends on the masses of the bodies (Gladman 1993). Thus - despite the results of the previous section - we can expect that the embryos eventually can achieve a phase of mutual scattering.

To explore the outcome of an instability phase in the vicinity of the planet trap, we have done a new series of three simulations, in which the inner embryo $\left(2 M_{\oplus}\right)$ is placed at the trap, and the outer one $\left(1 M_{\oplus}\right)$ is placed at $a \sim 1.17$, close enough to the inner embryo to be unstable from the beginning of the simulation. The initial eccentricity and inclination of the outer embryo are again of order $10^{-3}$ and $10^{-2}$ radians, respectively.

An example evolution from one of these simulations is illustrated in Fig. 6. At the first close encounter, the embryos acquire eccentric orbits, that are much more separated in semi major axis than the initial ones. Then, there is a competition between two processes: the damping of the eccentricities of the embryos exerted by the disk (Tanaka \& Ward 2004) and the excitation of the eccentricities due to the repeated mutual encounters between the embryos. The first process (eccentricity damping) is more effective. Thus, the eccentricities of both embryos decay, and the objects become dynamically decoupled (no close encounters are possible any more). The outer embryo starts to suffer an inward type I migration, until it is trapped into a mean motion resonance (the 6:7 in this case) which, as in the previous section, increases the eccentricity up to a new, equilibrium value. A stable configuration is achieved, characterized by an orbital separation larger than the one from which the simulation was started (the final semi major axis of the outer embryo stabilizes at 1.26 , while it started at 1.17). 


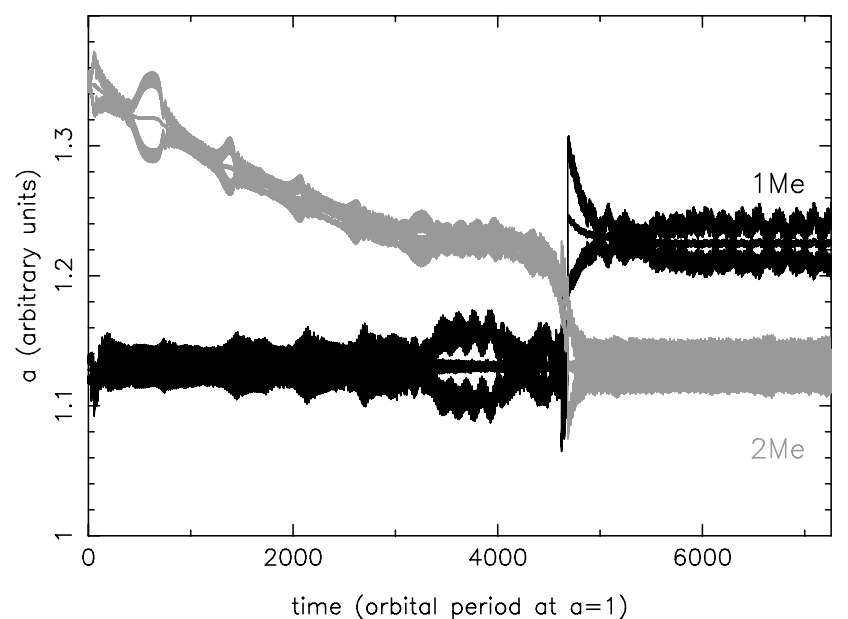

Fig. 7. As in the left panel of Fig. 3, but for reversed values of the masses.

This kind of evolution is common to two out of three of our simulations, and suggests that embryos can increase their orbital separation as they grow, through short instability phases of mutual scattering, followed by eccentricity damping and resonance trapping. The situation is similar to that found in the simulations of runaway growth of embryos in particle disks (Kokubo \& Ida 1996), where it was also noticed that the mutual orbital separation of the embryos increases with the embryos' masses. However, in one of our simulations the evolution is different. The outer, lighter embryo is scattered inward, into the inner part of the disk, as expected by Terquem \& Papaloizou (2007), where it will be free to migrate towards the central star. In this case the planet trap retains only the more massive embryo.

In all simulations, the scattering events excite the inclination of the more massive embryo to $\sim 0.05$ radians and the inclination of the less massive embryo to $\sim 0.1$ radians. After each scattering event, the inclination is damped exponentially, as imposed by Eq. (2).

Because they involve some scattering, we present in this section also simulations of two embryos initially on separated orbits as in Sect. 4, but in which the more massive embryo is originally the outer one. We have done three simulations of this kind, with masses of the embryos pairs equal to 1 and $2 M_{\oplus}, 1$ and $2.5 M_{\oplus}, 5$ and $10 M_{\oplus}$. An example evolution is given in Fig. 7, taken from the first of these simulations, but common to all of them. Because it is less massive, the inner embryo cannot prevent the migration of the outer embryo by trapping it in an outer mean motion resonance. One could expect that the inner embryo is pushed inwards from the trap by the outer embryo. However, this does not occur, because the corotation torque at the planet trap is stronger than the Lindblad torque felt by the outer embryo (see Fig. 1 in Masset et al. 2006a), despite the latter being more massive. What happens is that the orbit of the inner embryo tends to become eccentric when the outer one is trapped into a resonance. Eventually the resonant configuration is broken, and the two embryos have close encounters with each other. A possible outcome of the close encounter phase is a switch of position between the two bodies, with the more massive embryo taking the position of the lighter one at the planet trap. At this point, the evolution is analogous to that illustrated in Fig. 6. In some cases, the inner lighter embryo is scattered into the inner disk, while the outer, more massive embryo is scattered onto an orbit with large semi major axis and eccentricity. The eccentricity of the orbit of the more massive embryo is eventually damped and type I

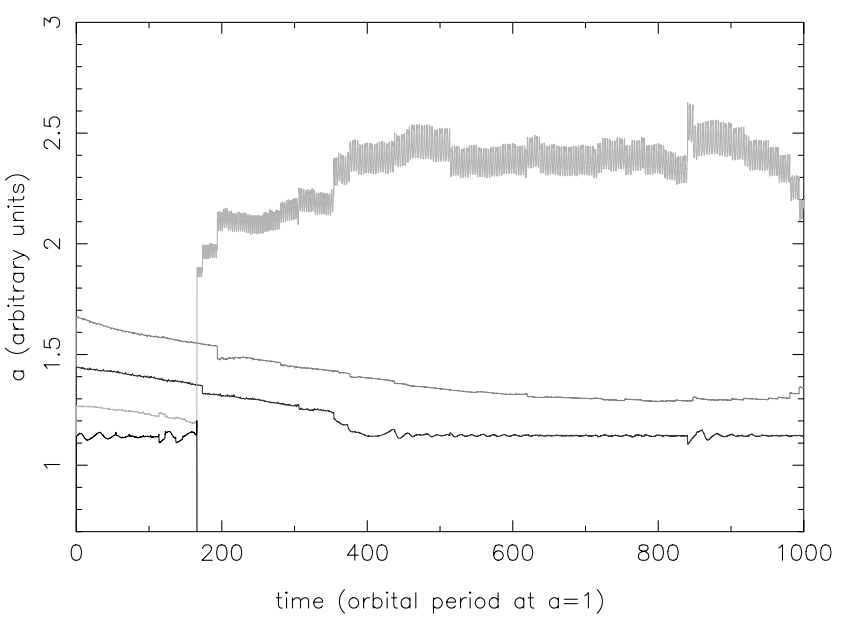

Fig. 8. The evolution of the semi major axes of 4 cores with masses equal to $5,10,14$ and $14 M_{\oplus}$ for the initially innermost to the outermost one. The $5 M_{\oplus}$ core is initially placed at the planet trap. The system is unstable and evolves to a configuration where one of the most massive cores takes the position at the trap.

migration brings it into the planet trap. In none of our three simulations we have observed a stable configuration with the lighter embryo at the planet trap and the more massive embryo at larger distance from the star.

Therefore, these results suggest that stable, non-migrating configurations of embryos, in which the the innermost object is the least massive one, should be rare. This is quite in contrast with the mass hierarchy of the cores of the giant planets of our solar system, where it seems (although the error bars are large) that the core of Jupiter is less massive than that of Saturn, which is in turn less massive than that of Uranus or Neptune (Guillot 1999). As a confirmation that a system of 4 cores with masses increasing with heliocentric distance is unlikely to be stable, we placed a $5 M_{\oplus}$ core at the planet trap $(a \sim 1.13)$, one core of $10 M_{\oplus}$ at $a \sim 1.27$ and two cores of $14 M_{\oplus}$ at $a \sim 1.45$ and $a \sim 1.67$ respectively. These core masses are consistent with those inferred for the current cores of the giant planets, given the large uncertainties. We did 2 simulations, with slightly different initial locations of the cores. Both showed similar evolutions (see Fig. 8 for one example). The second embryo pushed its way towards the planet trap, and eventually destabilized the inner embryo. The lightest embryo was scattered into the inner part of the disk, and the second lightest one was scattered outward, beyond the two most massive cores. One of the most massive cores eventually took the place at the planet trap.

If the current cores of the giant planets have indeed masses that increase with heliocentric distance, the experiments above suggest one of the two possibilities: (i) either the core of Saturn completed its growth after that Jupiter had already acquired a substantially massive atmosphere, and similarly for Uranus relative to Saturn or (ii) the core of Jupiter and (partially) that of Saturn have been eroded by convective motion in the massive atmospheres of these giant planets, and they were significantly more massive when they formed (so that the mass hierarchy that allows stability was respected).

\section{Dynamics of a system of multiple embryos}

The results of the previous sections show that a system with two planetary embryos tends to find a stable configuration in which the bodies are isolated from each other. However, the 


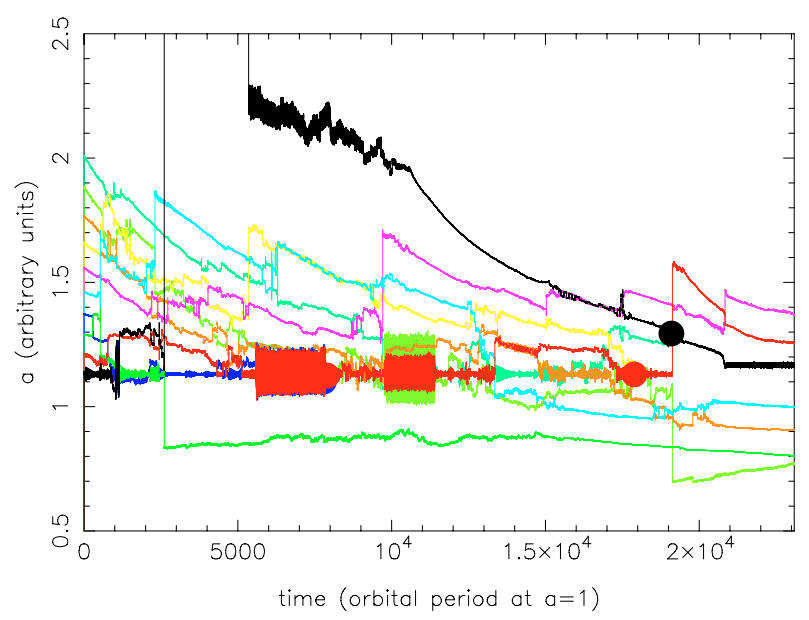

Fig. 9. The evolution of the semi major axes of 10 embryos of $1 M_{\oplus}$ each in the vicinity of the planet trap. The big red and black dots mark three collision events recorded in this simulation.

simulations with the 4 cores of the giant planets suggest that, with increasing number of bodies, the evolution can be more chaotic. Indeed, in more crowded systems of planetary embryos, we may expect that the dynamics is very different, as embryos can have more difficulties in finding a mutual orbital spacing that is large enough to ensure stability.

In this section we present a simulation with 10 embryos, each of one Earth mass. The inner one is placed at the planet trap at $a \sim 1.13$. The others are placed at increasing semi major axes, so that the initial orbital separation between two adjacent embryos is equal to 5 mutual Hill radii. The outermost planetary embryo is therefore placed at $a=2$. The initial eccentricities of all embryos are of order $10^{-3}$. The initial inclinations are of order $10^{-2}$ radians, and the longitudes of the ascending node are alternated by 180 degrees, relative to the neighboring embryo.

The evolution of the embryos is illustrated in Fig. 9. As expected, the system evolves much more chaotically than in the previous simulations. While the embryos at, or close to, the trap tend not to migrate, the outermost ones drift towards the star by type I migration. Some resonance trapping occurs, but these resonant phases do not remain stable for long time. Mutual scattering therefore dominates the dynamics and lasts for a much longer time than in the simulations with only two embryos presented in the previous sections. The embryo at the planet trap can be kicked out of the trap and its place can be taken by another embryo. There are episodes where two embryos share the same orbital semi major axis, in a relative tadpole motion (for instance the brown and light blue embryos between $t \sim 1000$ and $t \sim 2000$, or the brown and the red embryos between $t \sim 13500$ and $t \sim 15000$ ). Planets on mutual tadpole orbits have already been observed in the simulations by Cresswell \& Nelson (2006). We also find cases of embryos temporarily on a mutual satellite motion (the red and the blue embryos between $t \sim 5500$ and $t \sim 7500$, and the red and green embryos between $t \sim 10000$ and $t \sim 11500)$. These episodes are well visible, as they are characterized by large oscillations of the semi major axes of the concerned embryos. To our knowledge, this is the first simulation in which planets are found to trap each other in satellite motion. A satellite capture requires some kind of energy dissipation, presumably due to the interaction of the objects with the disk. Given the limited resolution of the grid used for the hydrodynamical calculation, we cannot exclude that the dissipation is artificially large, so that episodes of satellite motion might be much more rare in reality.

Due to the protracted phase of mutual encounters, three mutual collisions happen in this simulation. Two involve the embryo whose evolution is shown in red, and one the embryo whose evolution is shown in black. The collision events are marked by the big red and black dots respectively.

The evolution of the embryos calms down at $t \sim 21000$. At this time, 4 of the 7 surviving embryos have been kicked into the inner part of the disk $(a \leq 1)$. These embryos do not migrate significantly, because the surface density of the inner disk in our simulation is very low. If the inner disk had been more massive, these embryos would have started a monotonic inward type I migration, as in Cresswell \& Nelson (2006) simulations, and would have eventually been eliminated by a collision with the star. The black embryo (which has grown to $2 M_{\oplus}$ ) has taken the place at the planet trap. It seems to prevent the migration of the red embryo ( $3 M_{\oplus}$ ) and magenta embryo $\left(1 M_{\oplus}\right)$ outside of its orbit, similarly to what we have seen in the previous sections. Because the red embryo is the most massive, it might be possible that a continuation of this simulation could show an exchange between the orbits of the red and the black embryos, similarly to what happened to the red and magenta embryos at $t \sim 21000$.

Throughout the evolution, the inclinations of the embryos are excited by the scattering events, up to a few tenths of a radian, and then damp exponentially, until the next scattering event occurs. Figure 1 shows the evolution of the inclination of one of the embryos.

The comparison of this simulation with those of Cresswell \& Nelson (2006) is quite instructive. Here, because of the presence of the planet trap, which is an effective obstacle to migration, the embryos cannot find as easily a mutual stable orbital spacing. Thus the scattering phase is much more protracted in time, and collisions are more likely to occur. In addition, in Cresswell and Nelson simulations, all the embryos eventually drift towards the central star, while here three embryos are saved from migration, at the trap or just beyond it. Therefore, the planet trap appears to be an effective mechanism to prevent type I migration of some embryos, even in originally crowded systems. In these systems, the presence of the trap helps mutual collisions to occur and favors the growth of larger objects, even if not in the way envisioned in Masset et al. (2006a). Also, the trap acts like a filter, in the sense that, out of many initial embryos, only a few are allowed to survive in a stable non-migrating configuration. As long as the system is too crowded, stability cannot be achieved, and the number of embryos has to decay through collisions, ejections or injections into the inner disk. Eventually, a single planet trap helps the formation and the preservation of a few cores. This result may provide an explanation of why our solar system has only a limited number of giant planets, and no intermediate mass (of few Earth masses) planets in between them.

\section{Conclusions}

We have conducted a series of hydrodynamical simulations of the evolution of planetary embryos in a gas disk. The simulation scheme allows the embryos to evolve in three dimensions, whereas the disk is modeled with a 2 dimensional grid. A damping force on the inclinations of the embryos is applied. The disk has been built with a positive density gradient at $r \sim 1$, so that a "planet trap" (Masset et al. 2006a) is produced at $r \sim 1.1$.

Our simulations show that, because the planet trap is an obstacle to type I migration, a crowded system of embryos can not drift in concert towards the central star, preserving the relative 
mutual separations among the embryos. Instead, the system becomes violently unstable. Collisions between embryos become possible. Some embryos can be scattered into the inner part of the disk, where the planet trap mechanism is not effective any more to prevent their migration. The system stabilizes when the number of embryos behind the planet trap decreases down to a few. This may explain why our solar system has only a small number of giant planets.

A system with two (probably a few) embryos tends to find a stable, non-migrating configuration. The embryo at the planet trap restrains the other embryo(s) from migrating, through the action of its outer mean motion resonances, or (if its mass is large enough) through indirect perturbations. In this configuration, the embryos can continue to increase their mass in solid elements only by accreting small planetesimals, in a classical oligarchic growth regime. If they become too massive with respect to their orbital separation, they can acquire new, more separated orbits that are again stable and non migrating. This happens through a short phase of instability, during which mutual encounters emplace the objects onto orbits with more separate semi major axes and larger eccentricities, followed by aphase of eccentricity damping exerted by the disk. During the instability phases, the more massive embryo typically manages to take position at the planet trap, the lighter embryo(s) being emplaced on an orbit at larger semi major axis. Thus, the fact that in our solar system Jupiter is the giant-planet with the smallest core suggests that (i) either the completion of the cores of Saturn, Uranus and Neptune occurred after Jupiter had already acquired a substantial mass of gas in its envelope (possibly opening a gap and moving the planet trap to the outer edge of its gap), or (ii) that the the core of Jupiter (and partially that of Saturn) was originally more massive and was substantially eroded by convective motion in its atmosphere.

These results and considerations should not be limited to the planet trap scenario. Their validity should be more general. They should apply to any case in which some mechanism prevents an inner embryo to have a free type I migration.

Acknowledgements. We thank Alice Quillen and Willy Kley for useful, friendly comments. A.M. is grateful for support from the French programs PNP and OPV.

\section{References}

Alibert, Y., Mordasini, C., Benz, W., \& Winisdoerffer, C. 2005, A\&A, 434, 343 Balbus, S. A., \& Hawley, J. F. 1991, ApJ, 376, 214

Bergin, E., Calvet, N., Sitko, M. L., et al. 2004, ApJ, 614, L133

Calvet, N., Hartmann, L., Wilner, D., Walsh, A., \& Sitko, M. L. 2004, Debris Disks and the Formation of Planets, 324, 205

Calvet, N., D'Alessio, P., Watson, D. M., et al. 2005, ApJ, 630, L185

Chambers, J. E. 2001, Icarus, 149, 262

Chiang, E. I., \& Murray-Clay, R. A. 2007, [arXiv:0706.1241]

Cresswell, P., \& Nelson, R. P. 2006, A\&A, 450, 833

Cresswell, P., Dirksen, G., Kley, W., \& Nelson, R. P. 2007, [arXiv: 0707. 2225]

Ferreira, J., Dougados, C., \& Cabrit, S. 2006, A\&A, 453, 785

Forrest, W. J., Sargent, B., Furlan, E., et al. 2004, ApJS, 154, 443

Gammie, C. 1996, ApJ, 457, 355

Gladman, B. 1993, Icarus, 106, 247

Guillot, T. 1999, Science, 286, 72

Ilgner, M., \& Nelson, R. P. 2006a, A\&A, 445, 223

Ilgner, M., \& Nelson, R. P. 2006b, A\&A, 445, 223

Johnson, E. T., Goodman, J., \& Menou, K. 2006, ApJ, 647, 1413

Kokubo, E., \& Ida, S. 1996, Icarus, 123, 180

Kokubo, E., \& Ida, S. 1998, Icarus, 131, 171

Laughlin, G., Steinacker, A., \& Adams, F. C. 2004, ApJ, 608, 489

Lubow, S. H., \& Ogilvie, G. I. 2001, ApJ, 560, 997

Masset, F. 2000a, A\&A, 141, 165

Masset, F. S. 2000b, Disks, Planetesimals, and Planets, 219, 75

Masset, F. S., Morbidelli, A., Crida, A., \& Ferreira, J. 2006a, ApJ, 642, 478

Masset, F. S., D’Angelo, G., \& Kley, W. 2006b, ApJ, 652, 730

McNeil, D., Duncan, M., \& Levison, H. F. 2005, AJ, 130, 2884

Nelson, R. P. 2005, A\&A, 443, 1067

Nelson, R. P., \& Papaloizou, J. C. B. 2003, MNRAS, 339, 993

Nelson, R. P., \& Papaloizou, J. C. B. 2004, MNRAS, 350, 849

Ogihara, M., Ida, S., \& Morbidelli, A. 2007, Icarus, 188, 522

Paardekooper, S.-J., \& Mellema, G. 2006, A\&A, 459, L17

Papaloizou, J. C. B., Nelson, R. P., \& Snellgrove, M. D. 2004, MNRAS, 350, 829

Papaloizou, J. C. B., \& Szuszkiewicz, E. 2005, MNRAS, 363, 153

Piétu, V., Dutrey, A., Guilloteau, S., Chapillon, E., \& Pety, J. 2006, A\&A, 460, L43

Pollack, J. B., Hubickyj, O., Bodenheimer, P., et al. 1996, Icarus, 124, 62

Shakura, N. I., \& Sunyaev, R. A. 1973, A\&A, 24, 337

Steiner, E. 1996, The Chemistry Maths Handbook, Oxford

Stone, J. M., Ostriker, E. C., \& Gammie, C. F. 1998, ApJ, 508, L99

Tanaka, H., \& Ward, W. R. 2004, ApJ, 602, 388

Tanaka, H., Takeuchi, T., \& Ward, W. R. 2002, ApJ, 565, 1257

Terquem, C., \& Papaloizou, J. C. B. 2007, ApJ, 654, 1110

Turner, N. J., Sano, T., \& Dziourkevitch, N. 2007, ApJ, 659, 729

Ward, W. R. 1997, Icarus, 126, 261 